Pacific Journal of Mathematics

NECESSARY AND SUFFICIENT CONDITIONS FOR CERTAIN
HOMOLOGY 3-SPHERES TO HAVE SMOOTH $Z_{p}$-ACTIONS ERICA Flapa 


\title{
NECESSARY AND SUFFICIENT CONDITIONS FOR CERTAIN HOMOLOGY 3-SPHERES TO HAVE SMOOTH $\mathbf{Z}_{p}$-ACTIONS
}

\author{
ERICA FLAPAN
}

\begin{abstract}
We derive necessary and sufficient conditions for a broad class of homology 3-spheres, obtained as the gluing of two knot complements, to have $\mathbf{Z}_{p}$-actions.

We explore when a homology sphere, obtained as the gluing of two knot complements has any smooth periodic diffeomorphisms.
\end{abstract}

Introduction. Myers [6] has given examples of homology spheres with no P.L. involutions. He constructed these examples by gluing together particular types of knot complements. Initially, we construct different types of periodic homology spheres out of different types of periodic knots. One of our constructions yields homology spheres with orientation reversing involutions in a simpler manner than that of Siebenmann and Van Buskirk [8]. Next, we prove the necessity of our conditions and are then able to construct infinitely many non-periodic homology spheres. In addition, we establish conditions for such a homology sphere to be the branched cyclic cover of a knot.

We shall use the following notation. Let $K_{0}$ and $K_{1}$ be distinct knots in $S^{3}$. Let $N\left(K_{l}\right)$ be a tubular neighborhood of $K_{i}$. Let

$$
Q_{i}=S^{3}-\operatorname{Int} N\left(K_{\imath}\right) .
$$

Let $l_{l} \subseteq \partial Q_{i}$ be an oriented longitude for $Q_{l}$ in the sense that $l_{l}$ bounds a surface in $Q_{l}$. Let $m_{1} \subseteq \partial Q_{l}$ be an oriented meridian for $Q_{i}$ in the sense that $m_{\imath}$ bounds a meridional disk in $N\left(K_{i}\right)$. Let $M\left(K_{0}, K_{1}\right)$ be the irreducible homology 3-sphere obtained by gluing each $l_{i}$ on $Q_{i}$ to a $m_{j}$, on $Q_{j}, i \neq j$. Then $Q_{0} \cap Q_{1}=T$ a torus. We work throughout in the smooth category.

Our main result will be:

Theorem 3. Let $K_{0}$ and $K_{1}$ be distinct prime knots having Property $P$ and neither being a companion of the other. In addition suppose $K_{0}$ is not a torus knot or a cable knot. Let $p$ be a prime number, and $N_{i}$ a tubular neighborhood of $K_{i}$. 
1. $M\left(K_{0}, K_{i}\right)$ has an orientation reversing $\mathbf{Z}_{p}$-action $h$ iff $K_{i}$ is strongly positive amphericheiral and $K_{j}$ is strongly negative amphicheiral.

2. $M\left(K_{0}, K_{1}\right)$ has an orientation preserving $\mathbf{Z}_{p}$-action $h$ with $\operatorname{fix}(h) \cong S^{1}$ iff $K_{i}$ and $K_{j}$ are both strongly invertible.

3. $M\left(K_{0}, K_{1}\right)$ has a free $\mathbf{Z}_{p}$-action iff $K_{0}$ has a free $\mathbf{Z}_{p}$-action leaving a $(1, s)$ curve on $\partial N_{0}$ invariant and $K_{1}$ has a free $\mathbf{Z}_{p}$-action leaving an $(s, 1)$ curve on $\partial N_{1}$ invariant, for some $s$ which is knot a multiple of $p$.

We begin with some definitions.

DEFINITION 1. A knot is strongly negative amphicheiral if there is a smooth involution $g$ of $S^{3}$ which is orientation reversing and $g$ takes $K$ to itself with opposite orientation.

DefinITION 2. A knot is strongly positive amphicheiral if there is a smooth involution $g$ of $S^{3}$ which is orientation reversing and $g$ takes $K$ to itself with the same orientation.

DEFINITION 3. A knot is strongly invertible if there is an orientation preserving involution $g$ of $S^{3}$ and $g$ takes $K$ to itself with opposite orientation.

REMARK. If $h$ is an involution such that $h(K)=-K$ then $h$ fixes two points on $K$.

Definition 4. A knot $K$ has a free $\mathbf{Z}_{p}$-action if there is an order $p$ diffeomorphism of $S^{3}$, leaving $K$ invariant yet which is fixed point free.

Definition 5. A knot $K$ has a symmetry if there is a periodic diffeomorphism leaving $K$ invariant yet fixing a simple closed curve disjoint from $K$.

Definition 6. A $(p, q)$ curve on a torus is a curve wrapping around $p$ times longitudinally and $q$ times meridionally.

Definition 7. fix $(h)$ shall denote the fixed point set of $h$.

We begin to construct periodic homology spheres by understanding the behavior of an action in a tubular neighborhood of the knot.

LEMMA 1. Let $K$ be a knot in $S^{3}$ with a tubular neighborhood $N$, and let $p$ be a prime number. Suppose $K$ has an orientation preserving, order $p$, 
diffeomorphism $h$ such that $h(N)=N$ and $h$ fixes some point of $N$. Then $h$ fixes 4 points of $\partial N$ and $h$ is an involution with $h(K)=-K$.

Proof. Since fix $(h) \neq \varnothing$, by Smith Theory fix $(h)$ is a circle. Assume fix $(h) \subseteq$ Int $N$. Now fix $(h)$ is not knotted by the Smith Conjecture [13]. But $K$ is knotted, so by Schubert [7] fix $(h)$ must have order zero in $N$. Let $D$ be a meridional disk of $N$ which misses fix $(h)$. Let $X$ be the orbit space of $N$ induced by $h$. Now apply Dehn's Lemma in $X$ to modify $D$ so that $h^{i}(D) \cap D=\varnothing$ for all $i<p$. Let $B$ be the component of $N-\bigcup_{i=0}^{p-1} h^{i}(D)$ which contains fix $(h)$. Let $S$ be the 2-sphere bounding $B$. Now $h(B)=B$ so $h(S)=S$. Now fix $(h) \cap S=\varnothing$ so by Smith Theory for $S^{2}, h$ must be orientation reversing. But this is not possible, since $h$ is orientation preserving. Thus fix $(h) \subseteq$ Int $N$. So fix $(h) \cap \partial N \neq \varnothing$. Let $r$ be the number of points in fix $(h) \cap \partial N$. Let $Y$ be the orbit space of $\partial N$ induced by $h$. Then $\partial N$ is the $p$-fold branched cover of $y$ with $r$ branch points. So by the Riemann-Hurwitz formula $p \chi(Y)=r(p-1)$. Now $Y$ is an orientable 2 -manifold and $r(p-1)>0$. So $Y$ is a 2 -sphere. Now $2 p=r(p-1)$. Thus $(p-1)$ divides 2 or $p$. In other words $p-1=2$ or $p-1=1$. If $p=3$ then $r=3$. But $r$ is the number of times fix $(h)$ crosses $\partial N$. Hence $r$ must be even, since $\partial N$ separates. Therefore $p=2$, so $r=4$. Now $h$ takes a meridional disk of $N$ to a meridional disk, and a Seifert surface to a Seifert surface. Thus $h(l)$ is isotopic to $\pm l$, where $l$ is a longitude. Let $A$ be an arc in $Y$ connecting two branch points. Let $c$ be the complete lift of $A$ to $\partial N$. Then $c$ is a simple closed curve in $\partial N$ such that $h(c)=-c$. Now since $h$ is orientation preserving $h(l)$ must in fact be isotopic to $-l$. Finally $h(K)=-K$ since by hypothesis $h(K)= \pm K$.

LEMMA 2. Let $K$ be a knot in $S^{3}$ with a tubular neighborhood $N$, and let $p$ be a prime number. Suppose $h$ is a free $\mathbf{Z}_{p}$-action of $K$ such that $h(N)=N$. Then $h(c)=c$ for some $(r, 1)$ curve $c$, and $h\left(c^{\prime}\right)=c^{\prime}$ for some $(1, s)$ curve $c^{\prime}$, and $r s \equiv 1(p)$.

Proof. It follows from Hartley [3, Theorem 1.1 and the subsequent sentence] that $h$ leaves some $(r, 1)$ curve $c$ invariant.

Now by applying Dehn's Lemma in the orbit space of $N$ we can find a meridional disk $D$ such that $h^{i}(D) \cap D=\varnothing$. Let $m=\partial D$, and let $A^{\prime}$ be one component of $\partial N-\bigcup_{i=0}^{p-1} h^{i}(m)$. Let $a^{\prime}$ be an arc in $A^{\prime}$ from some point $x^{\prime} \in m$ to $h^{j}\left(x^{\prime}\right)$ such that $a^{\prime}$ goes less than once around $A^{\prime}$. Define $c^{\prime}=\bigcup_{i=0}^{p-1} h^{i}\left(a^{\prime}\right)$. Then $c^{\prime}$ is a $(1, s)$ curve. 
The essential intersection of $c$ and $c^{\prime}$ must consist of $r s-1$ points. Now $h$ permutes these points since $h(c)=c$ and $h\left(c^{\prime}\right)=c^{\prime}$. Thus $p$ divides $r s-1$. So $r s \equiv 1(p)$.

THEOREM 1. Let $K_{0}$ and $K_{1}$ be knots in $S^{3}$, and let $p$ be a prime number.

1. If $K_{0}$ is strongly positive amphicheiral, $K_{1}$ is strongly negative amphicheiral, and both knots are prime, then $M\left(K_{0}, K_{1}\right)$ has an orientation reversing involution $h$ with $\operatorname{fix}(h) \cong S^{0}$.

2. If $K_{0}$ and $K_{1}$ are both strongly invertible then $M\left(K_{0}, K_{1}\right)$ has an orientation preserving involution $h$ with $\operatorname{fix}(h) \cong S^{1}$.

3. If $K_{0}$ has a free $\mathbf{Z}_{p}$-action leaving $a(1, s)$ curve invariant and $K_{1}$ has a free $\mathbf{Z}_{p}$-action leaving an $(s, 1)$ curve invariant, then $M\left(K_{0}, K_{1}\right)$ has an orientation preserving $\mathbf{Z}_{p}$-action $h$ which is fixed point free.

Proof. 1. Let $N_{i}$ be a tubular neighborhood of $K_{i}$ such that $h_{i}\left(N_{i}\right)=N_{t}$ where $h_{l}$ is an orientation reversing involution with $h_{0}\left(K_{0}\right)=+K_{0}$ and $h_{1}\left(K_{1}\right)=-K_{1}$. By Smith Theory, since the $h_{l}$ are orientation reversing, fix $\left(h_{i}\right) \cong S^{0}$ or $\operatorname{fix}\left(h_{i}\right) \cong S^{2}$. Suppose fix $\left(h_{i}\right) \cong S^{2}$. Then by Smith Theory for $K_{i}, h_{\imath}$ fixes either zero or two points of $K_{i}$. Now $K_{i}$ cannot be contained in one component of $S^{3}-\operatorname{fix}\left(h_{i}\right)$ since $h_{l}$ must trade these components. Thus fix $\left(h_{l}\right) \cap K_{i}$ consists of two points. Hence $K_{i}$ is composite, contrary to hypothesis. So $\operatorname{fix}\left(h_{i}\right) \cong S^{0}$ for both $i$. Now we can assume we have picked $N_{i}$ such that $\operatorname{fix}\left(h_{i}\right) \cap \partial N_{i}=\varnothing$ for both $i$. Thus $h_{\imath} \mid \partial N_{i}$ covers its induced orbit space, which must be a Klein bottle. So $h_{0} \mid \partial N_{0}$ and $h_{1} \mid \partial N_{1}$ are equivalent actions. Let $l_{i}$ be a longitude and let $m_{l}$ be a meridian for $N_{l}$. Now $h_{i}$ takes a meridional disk to a meridional disk and a Seifert surface to a Seifert surface. So $h_{i}\left(m_{l}\right)$ is isotopic to $\pm m_{l}$ and $h_{t}\left(l_{l}\right)$ is isotopic to $\pm l_{i}$. Now since $h_{0}\left(K_{0}\right)=+K_{0}$ we must have $h_{0}\left(l_{0}\right)$ $\sim+l_{0}$, and since $h_{0}$ is orientation reversing $h_{0}\left(m_{0}\right) \sim-m_{0}$. Similarly $h_{1}\left(K_{1}\right)=-K_{1}$ implies that $h_{1}\left(l_{1}\right) \sim-l_{1}$, and so $h_{1}\left(m_{1}\right) \sim+m_{1}$ since $h_{1}$ is orientation reversing. Let $Q_{t}=S^{3}-\operatorname{Int}\left(N_{t}\right)$. Now we can glue $Q_{0}$ to $Q_{1}$ along their boundaries longitude to meridian to obtain $M\left(K_{0}, K_{1}\right)$; and $M\left(K_{0}, K_{1}\right)$ has an orientation reversing involution $h$ where $h \mid Q_{l}=h_{l}$. By Smith Theory for homology 3-spheres, fix $(h) \cong S^{2}$ or $\operatorname{fix}(h) \cong S^{0}$. But $\operatorname{fix}(h)=\operatorname{fix}\left(h_{0} \mid Q_{0}\right) \cup \operatorname{fix}\left(h_{1} \mid Q_{1}\right)$; thus fix $(h)$ cannot be $S^{2}$. So fix $(h) \cong S^{0}$ as desired.

2. Suppose $K_{0}$ and $K_{1}$ are strongly invertible. Let $h_{i}$ be an orientation preserving involution of $S^{3}$ such that $h_{l}\left(K_{l}\right)=-K_{i}$ and $h_{l}\left(N_{l}\right)=N_{l}$ for some tubular neighborhood $N_{l}$ of $K_{l}$. Then $h_{i}$ fixes two points on $K_{l}$ for each $i$. Now by Lemma $1 h_{t}$ fixes 4 points on $\partial N_{i}$, and so $\partial N_{t}$ is the 
two-fold branched cover of its orbit space, which is a 2-sphere. Now the action induced by $h_{i}$ on $\partial N_{i}$ is equivalent to reflecting both meridionally and longitudinally. Let $Q_{i}=S^{3}-\operatorname{Int}\left(N_{i}\right)$. Now glue $Q_{0}$ to $Q_{1}$ longitude to meridian to obtain $M\left(K_{0}, K_{1}\right)$; and $M\left(K_{0}, K_{1}\right)$ will have an orientation preserving involution $h$ where $h_{i}\left|Q_{i}=h\right| Q_{i}$. Now by Smith Theory for homology spheres fix $(h) \cong S^{1}$ or fix $(h)=\varnothing$. Now fix $(h)$ includes the 4 points fixed on $\partial N_{i}$, thus $\operatorname{fix}(h) \neq \varnothing$. So $\operatorname{fix}(h) \cong S^{1}$.

3. Suppose each $K_{i}$ has a smooth free $\mathbf{Z}_{p}$-action $h_{i}$. Let $N_{i}$ be a tubular neighborhood of $K_{i}$ such that $h_{i}\left(N_{i}\right)=N_{i}$ and there is a $(1, s)$ curve $c_{0}$, and an $(s, 1)$ curve $c_{1}$ on $\partial N_{i}$ such that $h_{i}\left(c_{i}\right)=c_{i}$. Pick numbers $r_{0}$ and $r_{1}$ such that $\left(h_{i}^{r_{i}}\right)$ is just a $2 \pi / p$ rotation along $c_{i}$. Now glue the

$$
Q_{i}=S^{3}-\operatorname{Int}\left(N_{i}\right)
$$

together longitude to meridian attaching $c_{0}$ to $c_{1}$. Now $M\left(K_{0}, K_{1}\right)$ has an orientation preserving $\mathbf{Z}_{p}$-action $h$ where $h\left|Q_{i}=h_{i}^{r_{i}}\right| Q_{i}$. Also, $h_{i}^{r_{i}}$ is fixed point free since $p$ is a prime, so $h$ is fixed point free as well.

REMARKS. 1. The construction in the first case of Theorem 1 provides a simpler method of obtaining an irreducible homology 3-sphere with an orientation reversing involution than that of Siebenmann and Van Buskirk [8].

2. We can find knots satisfying the conditions of case 3 of Theorem 1 by lifting knots in the appropriate lens spaces.

Now that we are able to construct homology spheres with $\mathbf{Z}_{p}$-actions with different types of fixed point sets we want to know whether the conditions on knot pairs in Theorem 1 are, in fact, necessary. That is, if $M\left(K_{0}, K_{1}\right)$ has a $\mathbf{Z}_{p}$-action in what sense do $K_{0}$ and $K_{1}$ inherit the action? We show that with a few added hypotheses our conditions are necessary. The first hypothesis we will add is that one knot complement contains no essential annulus. According to Simon [9, Lemmas 2.1 and 2.2] if the knot is not a composite, torus or cable knot then its complement contains no essential annulus.

LEMMA 3. Suppose $K_{0}$ is a prime knot which is not a torus knot or a cable knot, and suppose $K_{1}$ is any knot. Let $Q_{i}$ be the closed complement of $K_{i}$. Then every incompressible torus in $M\left(K_{0}, K_{1}\right)$ is isotopic to one disjoint from $T=\partial Q_{i}$.

Proof. Let $T^{\prime}$ be an incompressible torus in $M$. Isotop $T^{\prime}$ so that $T$ and $T^{\prime}$ meet transversely in a minimal number of components. Suppose 
some component $J$ of $T \cap T^{\prime}$ bounds a disk $D^{\prime}$ in $T^{\prime}$. Pick $J$ to be innermost, i.e. $D^{\prime} \cap T=\partial D^{\prime}$. Since $T$ is incompressible, $J$ also bounds a disk $D$ in $T$. Now since $M\left(K_{0}, K_{1}\right)$ is irreducible, $D \cup D^{\prime}$ bounds a 3-ball $B$. By pushing $D^{\prime}$ across $B$ we can remove $J$ from $T \cap T^{\prime}$, thus contradicting minimality. So there is no component of $T \cap T^{\prime}$ which bounds a disk in $T^{\prime}$. Hence $T^{\prime} \cap Q_{0}$ consists of properly embedded incompressible annuli. But by Simon [9, Lemmas 2.1 and 2.2] any such annulus must be boundary parallel in $Q_{0}$. So again we could remove the boundaries of this annulus by an isotopy of $T^{\prime}$, and so contradict minimality. Thus $T \cap T^{\prime}=\varnothing$.

REMARK 3. Let $h$ be a periodic diffeomorphism of a 3-manifold $M$, then, as is well known, we can choose a Riemannian metric for $M$ which makes $h$ an isometry. (Take the average of the $h$-transforms of any Riemannian metric.)

LEMMA 4. Let $K_{0}$ be a prime knot other than a torus knot or a cable knot, and let $K_{1}$ be any knot. Let $Q_{i}$ be the closed complement of $K_{i}$. Let $h$ be a periodic diffeomorphism of $M\left(K_{0}, K_{1}\right)$. Then $T=\partial Q_{i}$ is isotopic to a surface $S$ such that either $h(S)=S$ or $h(S) \cap S=\varnothing$.

Proof. By Theorem 1.1 of Freedman, Hass and Scott [2] since $M\left(K_{0}, K_{1}\right)$ is $P^{2}$-irreducible there is a least area immersion $f: T \rightarrow M$ which is homotopic to the inclusion $i: T \rightarrow M$. Since $i$ is incompressible $f$ must also be incompressible. Now by Alexander duality, since $H_{1}\left(M\left(K_{0}, K_{1}\right)\right)=0$ every closed surface in $M\left(K_{0}, K_{1}\right)$ is two-sided. By [2, Theorem 5.1] $f$ is an embedding. Hence $f(T)$ is a two-sided least area incompressible embedded torus. Since $h$ is an isometry, $h(f(T))$ is also a two-sided least area incompressible embedded torus.

Since $T$ and $f(T)$ are homotopic incompressible surfaces in an irreducible 3-manifold, by Waldhausen [12, Corollary 5.5] $T$ and $f(T)$ are ambient isotopic. Thus we can apply Lemma 3 to $f(T)$ to conclude that $h(f(T))$ can be isotoped disjoint from $f(T)$. Now apply Theorem 6.2 of [2] to conclude that either $h(f(T))=f(T)$ or $h(f(T)) \cap f(T)=\varnothing$; and let $S=f(T)$.

DeFinITION 8. If $h$ is a periodic diffeomorphism of $M\left(K_{0}, K_{1}\right)$ and $h\left(Q_{l}\right)=Q_{l}$, then we say $h$ is "good".

THEOREM 2. Suppose $K_{0}$ and $K_{1}$ are distinct prime knots having property $P$ and neither is a companion of the other. In addition suppose $K_{0}$ is 
not a torus knot or a cable knot. Then if $M\left(K_{0}, K_{1}\right)$ has a periodic diffeomorphism $h$ then $M\left(K_{0}, K_{1}\right)$ has a good diffeomorphism $h^{\prime}$ which is conjugate to $h$.

Proof. By the conditions on $K_{0}$ and $K_{1}$ we can apply Lemma 4 to get an isotopy $f_{t}$ of $M\left(K_{0}, K_{1}\right)$ such that $f_{0}$ is the identity and either $h\left(f_{1}(T)\right)=f_{1}(T)$ or $h\left(f_{1}(T)\right) \cap f_{1}(T)=\varnothing$. Let $h^{\prime}=f_{1}^{-1} \circ h \circ f_{1}$. Then $h^{\prime}$ has the same order as $h$ and either $h^{\prime}(T)=T$ or $h^{\prime}(T) \cap T=\varnothing$. Suppose $h^{\prime}(T) \cap T=\varnothing$. Recall, $Q_{i}$ is the closed complement of $K_{l}$ in $S^{3}$. Assume $h^{\prime}(T) \subseteq \operatorname{Int}\left(Q_{0}\right)$. If $h^{\prime}\left(Q_{0}\right) \subseteq \operatorname{Int}\left(Q_{0}\right)$ then

$$
Q_{0}=\left(h^{\prime}\right)^{p}\left(Q_{0}\right) \subseteq \operatorname{Int}\left(Q_{0}\right) \text {. }
$$

This contradiction implies that $h^{\prime}\left(Q_{0}\right) \nsubseteq \operatorname{Int}\left(Q_{0}\right)$ and hence

$$
h^{\prime}\left(Q_{1}\right) \subseteq \operatorname{Int}\left(Q_{0}\right) \subseteq S^{3},
$$

since $h^{\prime}\left(Q_{1}\right) \neq Q_{0}$ by Property P. Now $h^{\prime}(T)$ is essential in $M\left(K_{0}, K_{1}\right)$ so it is essential in $Q_{0}$ by Waldhausen [11, satz 1.9]. Let $V$ be the component of $S^{3}-h^{\prime}(T)$ containing $K_{0}$. Thus

$$
V \cup h^{\prime}\left(Q_{1}\right)=S^{3} \text { and } V \cap h^{\prime}\left(Q_{1}\right)=h^{\prime}(T) .
$$

Hence $V$ must be a solid torus. Let $J$ be the core of $V$. Then $J$ is isotopic to $K_{1}$, since $K_{1}$ has Property P. Now since $h^{\prime}(T)$ is essential in $Q_{0}$, we must have either $K_{1}$ is a companion of $K_{0}$ or $K_{1}$ is $K_{0}$ itself. Either case contradicts our hypotheses. We now use the same argument to show that $h^{\prime}(T) \nsubseteq \operatorname{Int}\left(Q_{1}\right)$. Thus $h^{\prime}(T)=T$. Now since both knots have Property P, $h^{\prime}\left(Q_{i}\right)=Q_{i}$.

\section{REMARKS.}

4. Since $l_{i}$ bounds a Seifert surface in $Q_{\imath}, h\left(l_{i}\right)$ must also. So on $T$, $h\left(l_{i}\right)$ is isotopic to $\pm l_{i}$. But since the $l_{i}$ are identified with the $m_{j}$, for $i \neq j, h\left(m_{j}\right)$ is isotopic to $\pm m_{i}$.

5 . By the above remark we can interpret the conclusion of Theorem 2 as saying that if $M\left(K_{0}, K_{1}\right)$ has a period $p$ diffeomorphism $h$, then $K_{0}$ and $K_{1}$ each have a period $p$ diffeomorphism $h_{i}$.

LemMa 5. Let $K$ be a knot in $S^{3}$ with a tubular neighborhood $N$ and let $p$ be a prime number. Suppose $h$ is a symmetry of $K$ such that $h(N)=N$; and that for some $(r, s)$ curve $c$ on $\partial N, r \neq 0, h(c)=c$. Then $s$ is a multiple of $p$.

Proof. By Edmonds and Livingston [1, Corollary 2.2] $K$ bounds a Seifert surface which is invariant under $h$. By intersecting this surface with 
$\partial N$ we can find a longitude $l$ such that $h(l)=l$. Now $c$ and $l$ must intersect essentially in $s$ points; and $h$ permutes these essential intersections. Thus $p$ must divide $s$.

Theorem 3. Let $K_{0}$ and $K_{1}$ be distinct prime knots having Property $P$ and neither being a companion of the other. In addition suppose $K_{0}$ is not a torus knot or a cable knot. Let $p$ be a prime number. Then:

1. $M\left(K_{0}, K_{1}\right)$ has an orientation reversing $\mathbf{Z}_{p}$-action $h$ iff $K_{i}$ is strongly positive amphicheiral and $K_{j}$ is strongly negative amphicheiral.

2. $M\left(K_{0}, K_{1}\right)$ has an orientation preserving $\mathbf{Z}_{p}$-action $h$ with $\operatorname{fix}(h) \cong S^{1}$ iff $K_{i}$ and $K_{j}$ are both strongly invertible.

3. $M\left(K_{0}, K_{1}\right)$ has a free $\mathbf{Z}_{p}$-action iff $K_{0}$ has a free $\mathbf{Z}_{p}$-action leaving a $(1, s)$ curve, on the boundary of a tubular neighborhood, invariant; and $K_{1}$ has a free $\mathbf{Z}_{p}$-action leaving an $(s, 1)$ curve, on the boundary of a tubular neighborhood, invariant, and $s \neq 0(p)$.

Proof. We have already established the sufficiency of the conditions in Theorem 1. We now establish necessity. Let $h$ be an order $p$ diffeomorphism of $M\left(K_{0}, K_{1}\right)$. By Theorem 2 we can assume that $h_{i}\left(Q_{i}\right)=Q_{i}$, where $Q_{i}$ is the closed complement of $K_{i}$. By Remarks 4 and 5 each $K_{l}$ has an order $p$ diffeomorphism $h_{i}$ such that $h_{i}\left|Q_{i}=h\right| Q_{i}$.

Case 1. If $h$ is orientation reversing. Then since $p$ is a prime, in fact $p=2$. By Remark $4 h\left(l_{i}\right)$ is isotopic to $\pm l_{i}$ on $T$. Now $l_{i}$ is identified with $m_{j}$, where $m_{j}$ is a meridian for $Q_{j}$. Since $h$ is orientation reversing if $h\left(l_{i}\right)$ is isotopic to $+l_{i}$ then $h\left(m_{i}\right)$ is isotopic to $-m_{i}$. Thus $h\left(l_{j}\right)$ is isotopic to $-l_{j}$. Hence $h\left(K_{i}\right)=+K_{i}$ but $h\left(K_{j}\right)=-K_{j}$. So $K_{i}$ is strongly positive amphicheiral, whereas $K_{j}$ is strongly negative amphicheiral.

Case 2. Now suppose $h$ is orientation preserving and fix $(h) \cong S^{1}$. First, assume fix $(h) \cap \partial Q_{i}=\varnothing$. Then for some $i$, say $i=0, \operatorname{fix}(h) \subseteq Q_{i}$. Thus fix $\left(h_{1} \mid Q_{1}\right)=\varnothing$. Now if $h_{1}$ fixed any point of $N_{1}=S^{3}-\operatorname{Int}\left(Q_{1}\right)$ then by Lemma $1, h$ would fix points on $\partial Q_{1}$. Hence fix $\left(h_{1}\right)=\varnothing$. So $h_{1}$ is a free $\mathbf{Z}_{p}$-action on $K_{1}$. Now by Lemma 2 there is an $(s, 1)$ curve $c$ on $\partial Q_{1}$ such that $h_{1}(c)=c$. Now $c$ is also a $(1, s)$ curve on $\partial Q_{0}$ and $h_{0}(c)=c$. But $\operatorname{fix}(h) \subseteq Q_{0}$ so $h_{0}$ is a symmetry of $K_{0}$. Thus by Lemma $5 s$ is a multiple of $p$. This is impossible, so fix $(h) \cap \partial Q_{i} \neq \varnothing$. Now by Lemma 1 $h$ is an involution and both $K_{i}$ are strongly invertible.

Case 3. Here $h$ is a free $\mathbf{Z}_{p}$-action of $M\left(K_{0}, K_{1}\right)$. So as in Case 2 we can apply Lemma 1 to conclude that $h_{i}$ is a free $\mathbf{Z}_{p}$-action for both $i$. Now 
apply Lemma 2 to $h_{0}$, to get a $(1, s)$ curve $c$ on $\partial Q_{0}$ which is invariant under $h_{0}$. Now $c$ is also a $(s, 1)$ curve on $\partial Q_{1}$ and is thus invariant under $h_{1}\left|\partial Q_{1}=h_{0}\right| \partial Q_{0}$. Also by Lemma $1, s$ has a multiplicative inverse in $\mathbf{Z} / p \mathbf{Z}$ so $s \neq 0(p)$.

REMARK 6. Note, in case 1 , that fix $(h) \not \equiv S^{2}$ since the knots are prime. Hence by Smith Theory fix $(h) \cong S^{0}$.

Corollary 1. Let $K_{0}$ and $K_{1}$ be distinct prime knots having Property $P$ and neither being a companion of the other. In addition suppose $K_{0}$ is not a torus knot or a cable knot. If $M\left(K_{0}, K_{1}\right)$ is any finite cyclic branched cover with branch set $S^{1}$, then both $K_{i}$ are strongly invertible.

Proof. If $M\left(K_{0}, K_{1}\right)$ were a finite cyclic branched cover of a knot then $M\left(K_{0}, K_{1}\right)$ has a covering translation $f$ and $\operatorname{fix}(f) \cong S^{1}$. Let $h$ be $f$ raised to a power, if necessary, so that the order of $h$ is a prime. Now $f$ was orientation preserving so $h$ is orientation preserving. Now by Smith Theory since $\operatorname{fix}(f) \subseteq \operatorname{fix}(h)$ in fact $\operatorname{fix}(h) \cong S^{1}$. Now apply Case 2 of Theorem 3.

Definition 9. A knot is simple if its complement in $S^{3}$ contains no essential torus.

REMARK 7. A simple knot has no companions. So if the $K_{i}$ are simple non-torus knots, it is enough to assume $Q_{0} \not \equiv Q_{1}$ in the proof of Theorem 2, we do not actually need Property P. Hence also in Theorem 3.

COROLlaRY 2. Let $K_{0}$ and $K_{1}$ be simple knots with non-homeomorphic exteriors. Suppose at least one of the knots is non-amphicheiral, one is non-invertible, and one has no free $\mathbf{Z}_{p}$-action. Then $M\left(K_{0}, K_{1}\right)$ has no periodic diffeomorphisms.

Proof. Observe that a torus knot has free $\mathbf{Z}_{p}$-actions. So if $K_{0}$ is the knot with no free $\mathbf{Z}_{p}$-actions then $K_{0}$ is not a torus knot. Since the knots are simple they are prime, neither is a companion of the other, and neither is a cable knot. Hence we can apply Theorem 3.

This corollary easily provides examples of irreducible homology 3spheres with no smooth $\mathbf{Z}_{p}$-actions. We show how to get an infinite collection of such homology spheres. Let $K_{0}$ be the $5_{2}$ knot. Then $K_{0}$ is 
2-bridge so it is simple by Kawauchi [5] and is non-amphicheiral. The Alexander polynomial of $K_{0}$ is $\Delta_{K_{0}}(t)=-2 t^{2}+3 t-2$ so by Hartley [3] $K_{0}$ has no free $\mathbf{Z}_{p}$-actions. Now, if $q, r$ and $s$ are distinct odd numbers bigger than one then the pretzel knot $K(q, r, s)$ is non-invertible by Trotter [10]. Since it is prime and 3-bridge, by Kawauchi it is simple. So we let $K_{1}=K(q, r, s)$. Now let $n=q s+q r+r s$, then $K(q, r, s)$ has Alexander Polynomial

$$
\Delta_{K_{1}}(t)=t^{2}\left(\frac{n+1}{4}\right)-t\left(\frac{n+1}{2}\right)+\left(\frac{n+1}{4}\right) .
$$

By our conditions on $q, r, s$ we have $n \geq 71$ so $\Delta_{K_{0}} \neq \Delta_{K_{1}}$ and thus $Q_{0} \not \equiv Q_{1}$. Now for distinct $n$ the knots $K(q, r, s)$ will have distinct Alexander polynomials and hence their complements are non-homeomorphic. Let $M\left(K_{0}, K_{1}\right)$ be one such homology sphere and let $M\left(K_{0}, K_{1}^{\prime}\right)$ be another. Then since $5_{2}$ and $K(q, r, s)$ are simple $M\left(K_{0}, K_{1}\right)$ and $M\left(K_{0}, K_{1}^{\prime}\right)$ each have precisely one incompressible torus. Suppose $M\left(K_{0}, K_{1}^{\prime}\right) \cong M\left(K_{0}, K_{1}\right)$, then there is a homeomorphism

$$
h: M\left(K_{0}, K_{1}^{\prime}\right) \rightarrow M\left(K_{0}, K_{1}\right)
$$

taking the incompressible torus $T^{\prime}$ to the incompressible torus $T$. Now since $Q_{0} \not \equiv Q_{1}$ we must have $h\left(Q_{1}^{\prime}\right)=Q_{1}$ hence the $n$ for $K_{1}^{\prime}$ must be the same as the $n$ for $K_{1}$. So for the infinite collection of distinct $n$ we will get infinitely many different non-periodic homology 3-spheres.

\section{REFERENCES}

[1] A. L. Edmonds and C. Livingston, Group actions on fibered three-manifolds, to appear, Comment. Math. Helv.

[2] M. Freedman, J. Hass and P. L. Scott, Least area incompressible surfaces in 3-manifolds, Invent. Math., 71 (1983), 609-642.

[3] R. I. Hartley, Knots with free period, Canad. J. Math., 33 (1981), 91-102.

[4] J. Hillman, Links with infinitely many semi-free periods are trivial, preprint 1983.

[5] A. Kawauchi, The invertibility problem on amphicheiral excellent knots, Proc. Japan Acad., 55, Ser. A (1979), 399-402.

[6] R. Myers, Homology spheres which admit no P. L. involutions, Pacific J. Math., 941 (1981), 379-384.

[7] Schubert, H., Knoten und Vollringe, Acta Math., 90 (1953), 131-286.

[8] L. C. Siebenmann and J. M. Van Buskirk, Construction of irreducible homology 3-spheres with orientation reversing involution, Pacific J. Math., 106 (1983), 245-255.

[9] J. Simon, An algebraic classification of knots in $S^{3}$, Ann. of Math., (2) 97 (1973), $1-13$.

[10] H. F. Trotter, Non-invertible knots exist, Topology, 2 (1964), 275-280. 
[11] F. Waldhausen, Eine Klasse von 3-dimensionale, Mannigfaltigkeiten, I, II, Invent. Math., 3 (1967), 308-333; Ibid. 4 (1967), 87-117.

[12] F. Waldhausen, On irreducible 3-manifolds which are sufficiently large, Ann. of Math., (2) 87 (1968), 56-88.

[13] Proceedings of the 1979 Conference on the Smith Conjecture at Columbia University, to appear.

Received March 18, 1983.

RICE UNIVERSITY

Houston, TX 77251 



\section{PACIFIC JOURNAL OF MATHEMATICS EDITORS}

DONALD BABBITT (Managing Editor)
University of California
Los Angeles, CA 90024
CHARLES R. DEPrIMA
California Institute of Technology
Pasadena, CA 91125
R. FINN
Stanford University
Stanford, CA 94305

\author{
HERMANN FLASCHKa \\ University of Arizona \\ Tucson, AZ 85721 \\ RAMESH A. GANGOLLI \\ University of Washington \\ Seattle, WA 98195 \\ ROBION KIRBY \\ University of California \\ Berkeley, CA 94720 \\ C. C. Moore \\ University of California \\ Berkeley, CA 94720
}

Hugo Rossi

University of Utah

Salt Lake City, UT 84112

H. SAMELSON
Stanford University
Stanford, CA 94305

HAROLD STARK

University of California, San Diego

La Jolla, CA 92093

\section{ASSOCIATE EDITORS}
R. ARens
E. F. BECKENBACH (1906-1982)
B. H. NeUmanN
F. WOLF
K. YOSHIDA

\begin{tabular}{ll}
\multicolumn{2}{c}{ SUPPORTING INSTITUTIONS } \\
UNIVERSITY OF ARIZONA & UNIVERSITY OF OREGON \\
UNIVERSITY OF BRITISH COLUMBIA & UNIVERSITY OF SOUTHERN CALIFORNIA \\
CALIFORNIA INSTITUTE OF TECHNOLOGY & STANFORD UNIVERSITY \\
UNIVERSITY OF CALIFORNIA & UNIVERSITY OF HAWAII \\
MONTANA STATE UNIVERSITY & UNIVERSITY OF TOKYO \\
UNIVERSITY OF NEVADA, RENO & UNIVERSITY OF UTAH \\
NEW MEXICO STATE UNIVERSITY & WASHINGTON STATE UNIVERSITY \\
OREGON STATE UNIVERSITY & UNIVERSITY OF WASHINGTON
\end{tabular}

The Supporting Institutions listed above contribute to the cost of publication of this Journal, but they are not owners or publishers and have no responsibility for its content or policies.

\footnotetext{
Mathematical papers intended for publication in the Pacıfic Journal of Mathematıcs should be in typed form or offset-reproduced (not dittoed), double spaced with large margins. Please do not use built up fractions in the text of the manuscript. However, you may use them in the displayed equations. Underline Greek letters in red, German in green, and script in blue. The first paragraph must be capable of being used separately as a synopsis of the entire paper. In particular it should contain no bibliographic references. Please propose a heading for the odd numbered pages of less than 35 characters. Manuscripts, in triplicate, may be sent to any one of the editors. Please classify according to the scheme of Math. Rev ews, Index to Vol. 39. Supply name and address of author to whom proofs should be sent. All other communications should be addressed to the managing editor, or Elaine Barth, University of California, Los Angeles, California 90024.

There are page-charges associated with articles appearing in the Pacific Journal of Mathematics. These charges are expected to be paid by the author's University, Government Agency or Company. If the author or authors do not have access to such Institutional support these charges are waived. Single authors will receive 50 free reprints; joint authors will receive a total of 100 free reprints. Additional copies may be obtained at cost in multiples of 50 .
}

The Pacific Journal of Mathematics is issued monthly as of January 1966. Regular subscription rate: $\$ 190.00$ a year (5 Vols., 10 issues). Special rate: $\$ 66.00$ a year to individual members of supporting institutions.

Subscriptions, orders for numbers issued in the last three calendar years, and changes of address should be sent to Pacific Journal of Mathematics, P.O. Box 969, Carmel Valley, CA 93924, U.S.A. Old back numbers obtainable from Kraus Periodicals Co., Route 100, Millwood, NY 10546.

The Pacific Journal of Mathematics at P.O. Box 969, Carmel Valley, CA 93924 (ISSN 0030-8730) publishes 5 volumes per year. Application to mail at Second-class postage rates is pending at Carmel Valley, California, and additional mailing offices. Postmaster: Send address changes to Pacific Journal of Mathematics, P.O. Box 969, Carmel Valley, CA 93924.

PUBLISHED BY PACIFIC JOURNAL OF MATHEMATICS, A NON-PROFIT CORPORATION Copyright $\odot 1985$ by Pacific Journal of Mathematics 


\section{Pacific Journal of Mathematics}

\section{Vol. 117, No. 2 \\ February, 1985}

Robert Walter Bagley, Ta-Sun Wu and J. S. Yang, On a class of topological groups more general than SIN groups ............... 209

Bruce Alan Barnes, Algebraic elements of a Banach algebra modulo an ideal ...............................................219

Howard D. Fegan and Peter Gilkey, Invariants of the heat equation .......233

Erica Flapan, Necessary and sufficient conditions for certain homology

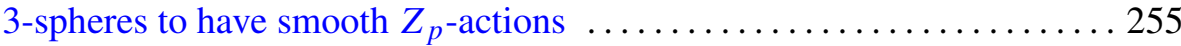

Philip R. Heath, Product formulae for Nielsen numbers of fibre maps . . . . 267

Derbiau Frank Hsu and A. Donald Keedwell, Generalized complete mappings, neofields, sequenceable groups and block designs. II ...... 291

Taqdir Husain, Orthogonal primitive idempotents and Banach algebras

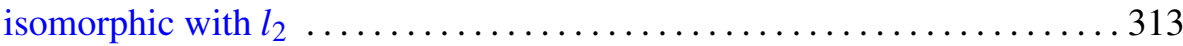

Gary M. Lieberman, Regularized distance and its applications . . . . . . . 329

William W. Menasco, Determining incompressibility of surfaces in alternating knot and link complements ............................ 353

Benjamin Muckenhoupt, Weighted reverse weak type inequalities for the Hardy-Littlewood maximal function $\ldots \ldots \ldots \ldots \ldots \ldots \ldots \ldots \ldots \ldots \ldots \ldots$

John Dacey O'Neill, Direct summands of direct products of slender

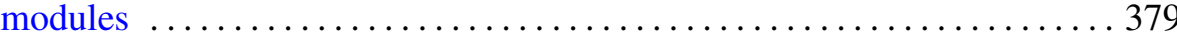

Andreas Rapp, Elimination of Malitz quantifiers in stable theories 387

Francisco José Ruiz, A unified approach to Carleson measures and $A_{p}$ weights

Hanamatagouda Pandappa Sankappanavar, Heyting algebras with dual

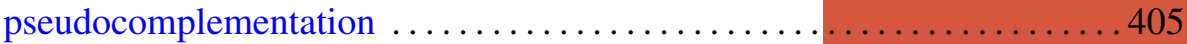

Annette Sinclair, Strong Carleman and strong uniform approximation . . . . 417

Masaaki Suzuki, The generalized Schwarz lemma for the Bergman metric

Brian Thorpe and Ludwig Tomm, Universal approximation by regular

weighted means 